\section{Hilft multidisziplinäre biopsychosoziale Reha bei chronischem Rückenschmerz?}

\author{
In dieser Studie sollte geklärt werden, ob sich multidisziplinär am biopsycho- \\ sozialen Modell orientierte Rehabilitation auf Schmerzreduktion, Behinde- \\ rung und Arbeitsausfällen bei Patienten mit chronischem Rückenschmerz \\ auswirkt.
}

\begin{abstract}
C hronischer Rückenschmerz weist eine hohe Prävalenzrate auf, ist für den größten Anteil der mit Behinderungen verbrachten Lebensjahre weltweit verantwortlich und mit hohen Gesundheitskosten verbunden. Da monotherapeutische Ansätze in klinischen Studien nur eine eingeschränkte Wirksamkeit zeigten, wurden multidisziplinäre Interventionen, die neben physischen auch psychische, edukative und arbeitsplatzbezogene Maßnahmen enthalten, entwickelt.

Der nach den Richtlinien der Cochrane Collaboration durchgeführte systematische Review und die Metaanalyse schloss randomisierte kontrollierte Studien zu Erwachsenen mit über drei Monaten anhaltendem Rückenschmerz ein. Als multidisziplinär galten Interventionen, die zusätzlich zur physischen psychische und/oder arbeitsbezogene Kom-
\end{abstract}

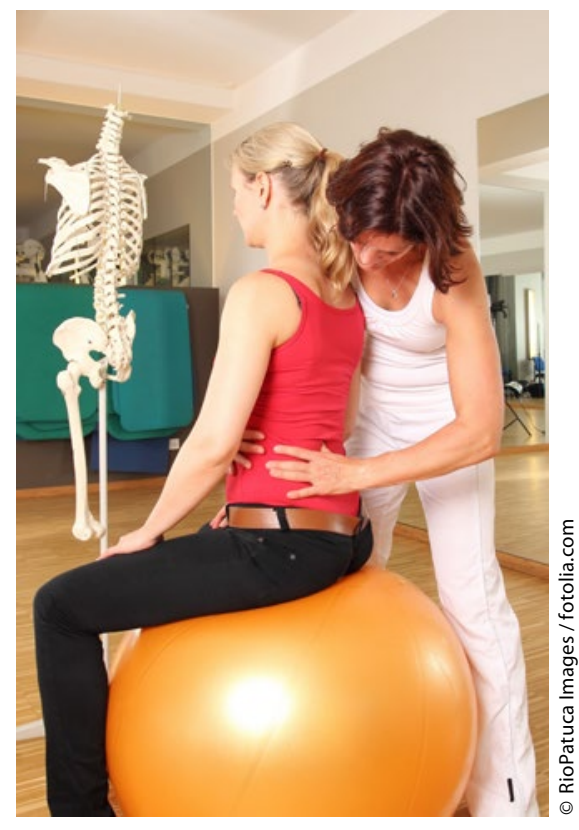

Die Reha sollte multidisziplinär und biopsychosozial ausgerichtet sein. ponenten umfassten. Kontrollinterventionen waren Standardversorgung, Physiotherapie, Operation oder Warteliste. Als primäre Endpunkte wurden langfristige Schmerzen, Behinderungen und Arbeitsausfälle gewählt.

41 Studien mit insgesamt 6.858 Teilnehmern wurden ausgewertet. $32 \%$ der Studien wiesen ein geringes Bias-Risiko auf, $79 \%$ beschrieben geeignete Randomisierungsmethoden und $56 \%$,allocation concealment". Eine Verblindung war nicht möglich. $63 \%$ berichteten vollständig über Ergebnisdaten, $39 \%$ wandten eine Intention-to-treat-Analyse an.

Multidisziplinäre Interventionen (MI) waren verglichen mit der Standardversorgung bezüglich Schmerzen $(n=821$; mäßige Studienqualität) und bezüglich Behinderungen ( $\mathrm{n}=722$; mäßige Studienqualität) wirksamer. Bezüglich Arbeitsausfällen ( $\mathrm{n}=1.360$; mäßige Studienqualität) zeigte sich kein Unterschied.

Verglichen mit Physiotherapie waren MI bezüglich Schmerzen ( $\mathrm{n}=872$; niedrige Studienqualität) statistisch nicht signifikant wirksamer, bezüglich Behinderung ( $\mathrm{n}=1.169$; mäßige Studienqualität) und bezüglich Arbeitsausfällen ( $\mathrm{n}=1.006$; mäßige Studienqualität) aber schon.

Verglichen mit Operationen zeigten sich keine signifikanten Unterschiede bezüglich Schmerzen, Behinderung und Arbeitsausfällen $(n=423$; niedrige Studienqualität).

Im Vergleich zur Warteliste waren MI bezüglich Schmerzen (sehr niedrige Studienqualität) und bezüglich Behinderung (niedrige Studienqualität) wirksamer.

Fazit: Patienten mit chronischem Rückenschmerz profitieren stärker von multidisziplinärer Rehabilitation als von Standardversorgung oder Physiotherapie in Bezug auf Schmerzen oder Behin- derung. Hinsichtlich Arbeitsausfällen waren MI wirksamer als Physiotherapie.

Kamper SJ, Apeldoorn AT, Chiarotto A et al. Multidisciplinary biopsychosocial rehabilitation for chronic low back pain: Cochrane systematic review and meta-analysis. BMJ 2015; 350: h444

\section{Kommentar von Dr. med.}

\section{Jessica T. Mattivi:}

Der Cochrane Review fasst die beste derzeit zur Verfügung stehende Evidenz zur Wirksamkeit von multidisziplinärer biopsychosozialer Rehabilitation bei chronischem Rückenschmerz zusammen. Multidisziplinäre Rehabilitation scheint demnach der üblichen Versorgung in Bezug auf Schmerzen und Behinderungen überlegen und im Hinblick auf Arbeitsausfälle wirksamer als alleinige Physiotherapie zu sein. Bei genauerer Betrachtung stellt sich allerdings heraus, dass die zwar statistisch signifikanten Unterschiede bezüglich einer Schmerzreduktion umgerechnet auf eine zehnstufige Skala nur einen halben Punkt betragen. Hinsichtlich Behinderung entspricht der Unterschied 1,5 Punkte auf der 24-stufigen Roland-Morris-Skala. Die Wahrscheinlichkeit, nach zwölf Monaten arbeitsfähig zu sein, ist für Teilnehmer an einem multidisziplinären Rehabilitationsprogramm etwas weniger als doppelt so hoch als bei alleiniger Physiotherapie.

Vergegenwärtigt man sich, dass die untersuchten biopsychosozialen Rehabilitationsprogramme kostenintensiv und mit erhöhtem Aufwand verbunden sind, so erscheint eine sorgfältige Abwägung zwischen Kosten und Nutzen solcher Interventionen dringend geboten. Da die Studienpopulation im Schnitt bereits seit mehr als einem Jahr an chronischen Schmerzen litt und ein großer Anteil bereits erfolglose Therapieversuche hinter sich hatte, sollten multidisziplinäre Rehabilitationsprogramme in erster Linie Patienten mit einer schlechten Prognose zugänglich gemacht werden.

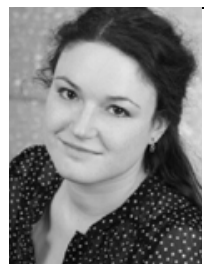

Dr. med. univ. Jessica T. Mattivi, MA

Klinik für Psychiatrie und Psychotherapie, Universitätsmedizin Mainz, E-Mail: jessica.mattivi@ unimedizin-mainz.de 